\title{
Le capital stratégique des communautés jamésiennes
}

\author{
Chakda Yorn, Karl Lussier et Paul Prévost ${ }^{1}$ \\ Coopérative de travail Niska
}

\section{Les communautés jamésiennes}

La région Nord-du-Québec a été creée en 1987 par décret gouvernemental. Elle couvre 860533 kilomètres carrés, soit $55 \%$ du territoire québécois, mais elle rassemble seulement $0,5 \%$ de la population. Ce territoire grandiose qui regorge de richesses naturelles abritait des autochtones depuis des millénaires. En 2006, elle regroupait moins de 14871 personnes et un peu plus de population de la Nation Crie, ce qui est une particularité indéniable.

La population établie plus au sud sur le territoire est formée surtout de gens arrivés massivement vers le milieu du $20^{\mathrm{e}}$ siècle pour exploiter les mines, la forêt et l'hydroélectricité. L'exploitation des ressources a donc favorisé l'établissement des communautés allochtones parmi les plus périphériques du Québec, soit Radisson, Valcanton, Villebois, Matagami, Lebel-sur-Quévillon, Chibougamau et Chapais. Leurs habitants sont appelés Jamésiens, ce qui permet de les distinguer de deux autres groupes de citoyens d'une grande importance dans la région Nord-du-Québec : les Inuits et les Cris. Ce trio socioculturel constitue une des spécificités de la région. Les conventions nordiques entre le gouvernement et les autochtones ainsi que les modifications subséquentes, dont la Paix des Braves, régulent les usages du territoire.

À maints égards, le Nord-du-Québec, et en particulier la Jamésie, représente un cas unique au monde dans les recherches sur le développement territorial : l'immensité du territoire, la structure spatiale des communautés qui sont très éloignées les unes des autres, la prépondérance des Nations autochtones et les conventions qui conditionnent les usages du territoire, l'organisation politique et administrative, la jeunesse des communautés et la concentration d'activités reliées à l'exploitation des ressources.

\section{Objectifs et démarche}

Cette étude s'est penchée sur l'analyse de la capacité des communautés locales jamésiennes à se développer de façon durable et à dégager des pistes d'actions intersectorielles permettant d'orienter et d'accroître la prise en charge des acteurs de la région. Une stratégie d'études de cas multiples a été déployée.

Chacune des sept communautés jamésiennes a fait l'objet d'une analyse détaillée et une analyse transversale a été menée afin de dégager les apprentissages les plus pertinents pour porter l'action. En tout, plus de 120 personnes ont été rencontrées en entrevue semi dirigée et en groupes de discussion. Les informants clés sont issus de divers horizons : maires et présidents de localités, professionnels municipaux, directeurs et professionnels des institutions publiques en économie, santé et services sociaux, jeunes, personnes âgées, intervenants dans les organismes communautaires. La triangulation des points de vue, la validation itérative ainsi qu'un forum regroupant les principaux acteurs de la région ont permis de baliser des pistes communes pour porter l'action.

\section{Quelques constats}

Pour constituer une communauté locale sur un territoire, une population doit pouvoir y travailler tout en étant capable d'absorber les contrecoups de la mouvance économique reliée à l'exploitation des ressources naturelles et aux autres industries. Plus le sentiment de stabilité économique est grand et l'offre d'emploi intéressante, plus les travailleurs seront atti- 
rés et viendront travailler sur ce territoire. Cette capacité de créer et de développer un bassin d'emploi alimente le projet de travail de la population.

Toutefois une communauté devrait pouvoir offrir plus qu'un projet de travail, elle devrait aussi offrir un projet de vie. En effet, une communauté locale nécessite également que la population locale, dont les travailleurs, ait envie de s'établir, d'acheter une maison, de fonder ou d'élever une famille, de s'épanouir personnellement, en plus de professionnellement, bref, d'avoir un projet de vie. La population s'investira si elle croit qu'elle peut générer un bien-être personnel dans le présent et qu'elle peut assurer le bien-être présent et futur de ses enfants. Sans projet de vie, il est plus difficile pour une population de se mobiliser et de se solidariser face aux difficultés. Sans projet de travail, elle ne peut survivre et encore moins prospérer. La figure 1 présente les cinq configurations particulières qui ont émergé de l'analyse pour caractériser le dynamisme des communautés de la Jamésie et ce, en fonction des projets de travail et de vie (deux axes) : carrefour de travailleurs, transition, chef-lieu, relance et revitalisation. Ces configurations facilitent la classification des communautés en termes de niveaux de vulnérabilité. Ainsi, Radisson, Valcanton et Villebois peuvent être situées parmi le niveau le plus élevé de vulnérabilité, tandis que Lebel-sur-Quévillon et Chapais sont de niveau moyen. Enfin, Chibougamau et Matagami, en raison de l'équilibre important qui existe entre le projet de vie et le projet de travail permettant une dynamique locale d'autodéveloppement, représentent un niveau plus faible de vulnérabilité.

\section{Figure 1 - Le dynamisme des communautés}

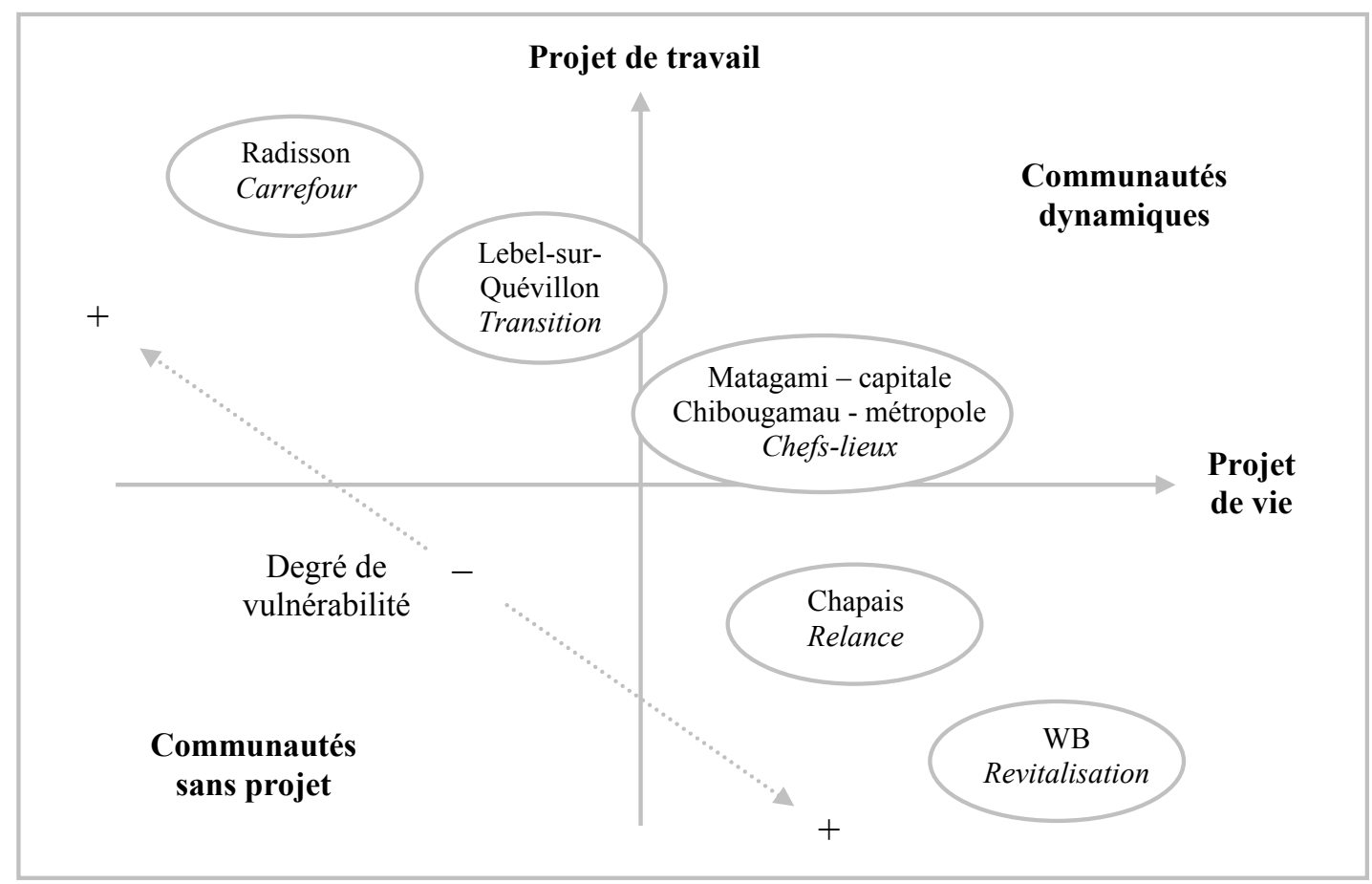

En plus des résultats d'analyse sur la dynamique locale, l'étude a fait ressortir cinq autres constats concernant les capacités de développement des communautés jamésiennes :

1) Les communautés ont des trajectoires distinctes basées sur l'exploitation de la terre (Valcanton et Villebois), des ressources naturelles (Chibougamau, Chapais, Matagami, Lebel-sur-Quévillon) et de l'hydroélectricité (Radisson).
2) La taille des différentes communautés locales est en général trop petite pour leur permettre de porter seules un plan de développement largement endogène de façon efficace et efficiente, d'où la nécessité de mettre en valeur le potentiel régional et de ses institutions publiques.

3) Outre les communautés rapprochées physiquement telles Chapais et Chibougamau ou Valcanton et Villebois, les communautés de la Jamésie n'ont 
à peu près pas de lien d'interdépendance entre elles. Même ces duos de communautés voisines auraient avantage à tisser des relations. Les espaces vécus se rejoignent donc peu en raison de la prédominance des relations nord-sud versus les relations est-ouest au sein de la région.

4) Les communautés ne sont pas unies entre elles par un destin commun car, pour l'instant, la région est plutôt utile que mentale, ce qui indique que la région comme communauté et comme culture régionale est une réalité très embryonnaire. De ce fait, mettre en commun le potentiel régional représente tout un défi et implique des innovations sociales ou des projets régionaux qui donnent une valeur ajoutée à chacune des communautés pour prouver d'abord l'utilité de la région et construire tranquillement la région mentale.

5) L'ensemble de la Jamésie regroupe des acteurs variés malgré la taille de population assez limitée. Les ressources et les entités politiques de la région sont relativement importantes en comparaison d'autres régions, ce qui laisse entrevoir des possibilités concrètes pour canaliser et gérer le développement.

\section{Le capital stratégique des communautés jamésiennes}

Le capital stratégique est un cadre de référence pour évaluer les atouts dont disposent une communauté et un territoire pour se développer. Ce cadre permet de définir l'état des lieux, soit les actifs physiques exceptionnels et les facteurs intangibles essentiels qu'une communauté peut activer. Ces atouts la rendent apte à acquérir d'autres ressources utiles et à saisir les opportunités afin d'influencer sa trajectoire de développement, faciliter sa propre prise en charge et la mâ̂trise de son développement.

Le capital stratégique est issu des autres formes de capitaux reconnus : le capital physique (incluant les ressources naturelles), le capital humain, le capital économique (incluant le capital financier), le capital social (incluant la culture) et le capital institutionnel. L'ensemble des capitaux de base réfère au patrimoine de la communauté et du territoire. La figure 2 illustre la notion de capital stratégique. On retrouve trois dimensions au concept de capital stratégique : les actifs stratégiques du territoire, le potentiel d'intervention et le potentiel de changement.

\section{Figure 2 - Conceptualisation du capital stratégique}

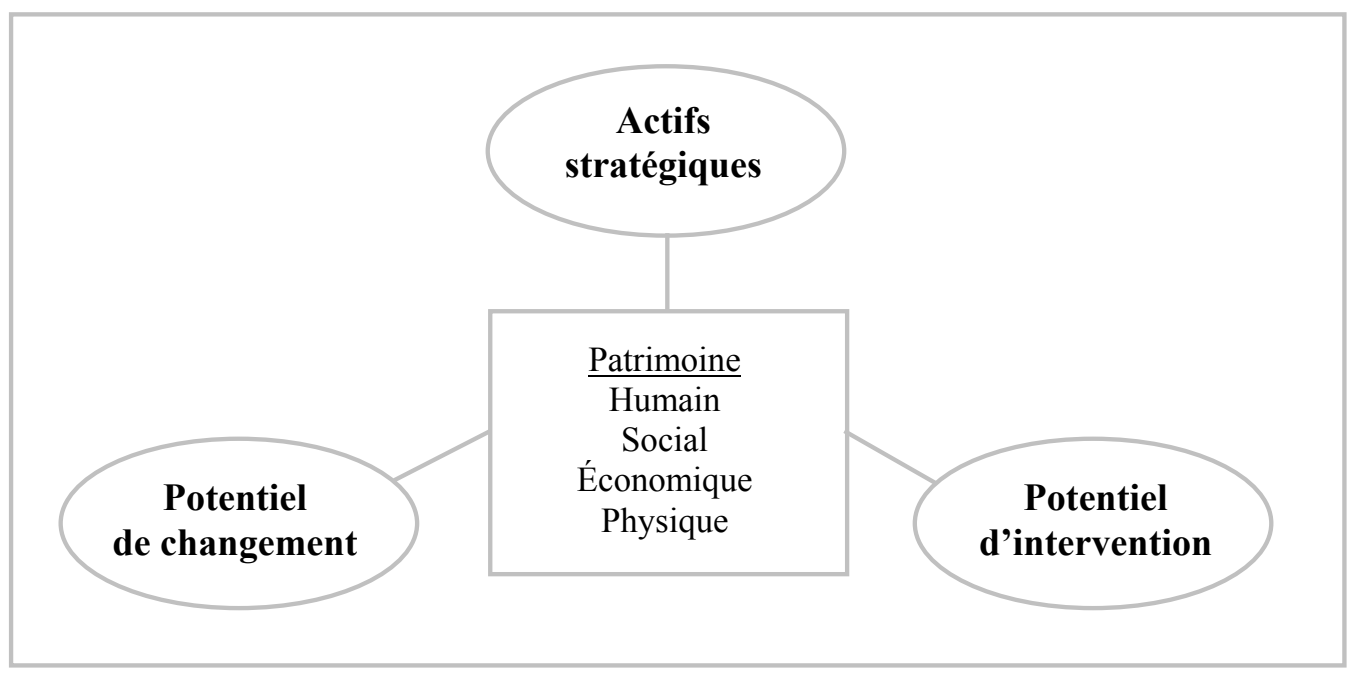

Les actifs stratégiques comprennent la partie la plus tangible du capital stratégique et font référence au patrimoine naturel, bâti et productif, offrant un potentiel de développement. Ils peuvent parfois constituer une valeur insoupçonnée. Ils peuvent être activés par la demande, par un entrepreneur visionnaire, un développement technologique, une conjoncture économi- que, politique et sociale favorable. Ainsi, que l'Égypte possède les grandes pyramides, que l'Alberta soit dotée de sables bitumineux ou que la Jamésie regorge de métaux, de forêt et d'eau, voilà qui illustre cette notion d'actifs d'exception. Ces actifs permettent de produire des avantages absolus et comparatifs sur le marché. 
Le potentiel d'intervention regroupe des éléments d'ordre humain, social et surtout organisationnel et caractérisent les capacités de gestion de la communauté et de ses acteurs. On y retrouve la gouvernance, la capacité citoyenne, le « communautéship » et l'entrepreneurship. Dans un premier temps, c'est à travers l'analyse des initiatives passées et des comportements des acteurs qu'on peut détecter la présence et caractériser ce potentiel d'intervention.

\section{Malgré le fait que les communautés locales vivent des problématiques similaires quant au capital humain, social, économique et physique, elles ne sont pas liées par une dynamique commune de développement.}

Le potentiel d'intervention repose beaucoup sur la notion de leadership, une réalité délicate et difficile à prendre en compte lors des interventions sur le terrain. Bien que fort utile, comme elle risque de qualifier ou de déqualifier des individus, elle est souvent une source de tension plutôt qu'un élément porteur. Dans une communauté, on préférera donc utiliser le concept de leadership partagé ou de « communautéship », un concept inventé par Henry Mintzberg. Celui-ci peut se définir comme un phénomène qui repose sur la contribution de plusieurs personnes ou organisations agissant selon leurs capacités dans un processus social et collectif de coopération pour le bien-être commun de la région et des communautés qui la composent. En effet, les spécificités des régions limitent la portée du leadership d'un seul individu capable de représenter toute la région, de façon légitime. La « communautéship » est aussi plus représentative de la variété des membres provenant des organismes et institutions souvent publiques d'une communauté ainsi que de la variété de leur travail effectif.

Le potentiel de changement représente la culture locale de développement d'une communauté, c'est-àdire ses valeurs et comportements qui facilitent le changement et la résolution de problèmes, et surtout la prise en charge du développement par la communauté. Le potentiel de changement intègre les notions de culture entrepreneuriale et d'innovation, d'ouverture sur le monde et au monde, de cohésion et de solidarité sociales et le sentiment d'appartenance.

L'étude a permis de souligner le rôle essentiel de la région sur le développement des communautés jamé- siennes. Or, le capital stratégique permettant à la région de jouer ce rôle se caractérise d'abord par la présence de ressources naturelles et financières d'envergure. Ces derniers atouts constituent des actifs exceptionnels au regard d'autres entités régionales au Québec ou ailleurs.

De plus, le capital stratégique de la région est composé de facteurs managériaux et politiques importants qui lui confèrent un certain potentiel d'intervention sur le développement de la région et des communautés locales. Ce potentiel se traduit par les rôles de leaders et d' " entrepreneurs institutionnels » que jouent les organismes régionaux: Municipalité de BaieJames, Conseil régional des élus, Société de développement de la Baie-James, Centre régional de santé et de services sociaux de la Baie-James, Commission scolaire de la Baie-James, Conseil régional des partenaires du marché du travail de la Jamésie, Direction régionale du ministère des Affaires municipales et des Régions, etc. Ces organismes constituent des leviers importants pour la région, car ils permettent de valoriser certaines ressources du territoire et d'acquérir d'autres ressources de l'extérieur étant donné qu'ils possèdent, notamment, un pouvoir politique significatif face au palier supérieur de gouvernement.

En outre, ces acteurs représentent, dans la région, un potentiel de changement, car ils participent à la création de liens entre les communautés locales, même si ces liens sont avant tout fonctionnels. En effet, ces liens ne semblent pas créer l'effet de proximité nécessaire à la création d'une région mentale, d'un espace vécu et partagé par l'ensemble des communautés. Cela leur permet de construire une région utile à l'ensemble des communautés. Car malgré le fait que les communautés locales vivent des problématiques similaires quant au capital humain, social, économique et physique, elles ne sont pas liées par une dynamique commune de développement. De plus, en raison d'un passé basé sur la grande entreprise organisatrice de la vie économique et sociale, on constate une faiblesse de l'esprit d'entreprise, de la solidarité et de l'identité régionales, représentant par le fait même un frein au développement.

En ce sens, le Comité de développement social de la Baie-James (CDSBJ) est aussi un acteur important sur la scène régionale, car il possède des leviers susceptibles de porter des actions en vue de renforcer la région mentale. Il est significatif parce que c'est un co- 
mité volontariste qui tente, peut-être pour la première fois, d'organiser des réflexions horizontales hors du cadre de leurs champs d'action habituels. Et malgré les difficultés, les composantes de ce comité, c'est-àdire les acteurs institutionnels régionaux, représentent un potentiel d'intervention signifiant dans la région jamésienne. Dans le contexte jamésien, il apparaît donc nécessaire de transformer les valeurs et comportements de la population pour donner à la communauté régionale et aux communautés locales la capacité d'impulser un changement de l'intérieur. C'est un travail de très longue haleine.

Les actifs exceptionnels, le potentiel d'intervention et le potentiel de changement synthétisent les éléments du capital stratégique régional qui nous sont apparus les plus significatifs dans une perspective de développement durable des collectivités en Jamésie. Si la région possède des capacités intéressantes à cet égard, les communautés locales aussi possèdent des atouts significatifs pour leur développement.

\section{Les pistes d'action régionales}

En conséquence, les actions régionales pour un développement durable devraient être orientées vers le renforcement du potentiel de changement de la région en s'appuyant sur le potentiel d'intervention de ses institutions. Pour ce faire, c'est un véritable projet d'innovation sociale qu'il faut mettre en place. Deux stratégies peuvent être proposées pour initier cette transformation :

1) construire une véritable culture régionale;

2) renforcer la gouvernance régionale sans brimer le principe de subsidiarité ${ }^{2}$ qui permet la marge d'action locale de chacune des communautés.

La construction de la culture régionale doit se traduire par des activités qui permettront, entre autres, de :

- développer une vision partagée des enjeux communs touchant le territoire et la population et, à cet effet, le Colloque sur le développement social de 2003 est un bon exemple;

- promouvoir l'histoire commune et conscientiser les gens à l'opportunité de construction quotidienne de cette histoire;

- générer des activités d'échange permettant de construire et de renforcer l'espace vécu (ex. orga- niser des occasions de sports, de loisirs, de culture ou d'affaires entre les communautés; créer des canaux de communication tels qu'une plateforme Internet jeunesse sur le site de la MBJ);

- initier et formuler au moins un projet collectif de développement dont les retombées constituent une valeur ajoutée pour la majorité ou pour toutes les communautés jamésiennes. Par exemple, une petite partie des retombées financières de l'entente avec Hydro-Québec pourrait servir à développer des projets territoriaux communs. La nouvelle Politique nationale de la ruralité (PNR) contient aussi des moyens additionnels qui pourraient servir de leviers à des projets régionaux de mobilisation;

- considérer les autochtones comme une condition sine qua none pour faire du co-développement. L'importance des autochtones est sans commune mesure avec d'autres régions du Québec. Avec 15466 personnes en 2005, les populations cries et naskapis ont déjà dépassé les Jamésiens et l'écart se creusera avec le temps. Toute stratégie devrait en tenir compte positivement, dépasser le stade des discours, intensifier et favoriser des projets territoriaux politiques et des projets spécifiques menant à des bénéfices mutuellement gagnants. Cela doit devenir naturel. Aussi, les Jamésiens se tournent automatiquement vers le Sud, alors que des avenues avec les Inuits au Nord s'avéreraient fort pertinentes à explorer.

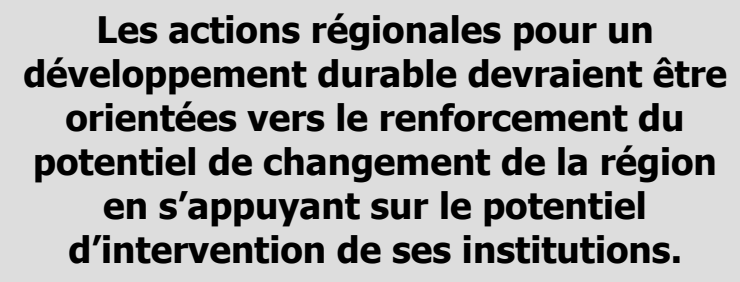

Le renforcement de la gouvernance régionale implique pour sa part de :

- valoriser le travail des leaders et des entrepreneurs régionaux pour susciter la solidarité et l'appui aux initiatives d'envergure pouvant représenter des retombées pour les communautés;

- favoriser la concertation et le partenariat entre les organismes par la mise sur pied et le soutien des initiatives intersectorielles. Le tout pourrait favoriser une « communautéship » qui renforce par le fait même la construction de la culture régionale; 
- favoriser la participation des individus à la réflexion sur les enjeux, les orientations et les décisions concernant l'ensemble de la population;

- considérer les autochtones comme une condition sine qua none pour faire du co-développement;

- multiplier des initiatives basées sur la formule des négociations avec le gouvernement ou avec les autochtones relève d'un rapport de force où la région est significative. Un précédent a été créé, comme l'illustre l'entente avec Hydro-Québec promettant des retombées régionales d'envergure. Il pourra aussi y avoir des retombées uniquement subrégionales mais pour lesquelles la région agit en tant que véritable palier de gouvernement régional dans les négociations.

Face aux enjeux identifiés et aux stratégies proposées, le CDSBJ est bien placé pour assumer un rôle de premier plan, car il possède non seulement la crédibilité mais aussi plus de leviers pour agir sur les éléments intangibles que sont les valeurs et les comportements de l'ensemble de la population jamésienne. Ainsi, son mandat devrait intégrer ces considérations dans son positionnement et ses actions futures. Enfin, en appuyant la mise sur pied de deux communautés stratégiques pour porter la réalisation de ces stratégies, le CDSBJ pourra porter un cran plus loin son implication stratégique dans le renforcement des capacités des communautés jamésiennes à se prendre en charge et à se développer de manière durable.

\section{Les pistes d'action locales}

Clairement, l'enjeu démographique est très prenant. Chacune des communautés jamésiennes a intérêt à développer des initiatives ou des projets de rétention, d'attraction et d'intégration de la population. Répondre à cet enjeu peut devenir un projet spécifique de développement local. Il peut être vu comme étant une initiative émergente d'une réflexion restreinte ou élargie qui met en valeur ou améliore les qualités intrinsèques et les opportunités du territoire afin de retenir la population actuelle et/ou d'attirer et d'intégrer des catégories de populations plus ou moins ciblées pour contrer un ou plusieurs éléments du cercle de dévitalisation territoriale. Les initiatives en ce sens se multiplient au Québec.

L'autre élément constant dans toute la région est le taux de rotation démographique, surtout dans la caté- gorie des professionnels. Souvent perçue comme un désavantage, la région et les communautés jamésiennes pourraient plutôt considérer cette rotation comme un avantage et se voir comme une " région tremplin » pour les professionnels qui ont la chance d'avoir eu des responsabilités importantes et de les avoir eues rapidement. Ils deviendront des ambassadeurs de la région si leur expérience a été enrichissante professionnellement, humainement et socialement.

\section{Chacune des communautés jamésiennes a intérêt à développer des initiatives ou des projets de rétention, d'attraction et d'intégration de la population.}

En sus de l'enjeu démographique, sur le plan local, et en fonction des enjeux et des potentialités de chacune des sept communautés, le CDSBJ et ses membres, en tant que mandataires de l'étude, auraient intérêt à promouvoir et à appuyer les pistes suivantes pour l'action locale. Ces pistes se situent surtout dans le domaine du développement social, comme le prévoyait le mandat de cette étude.

Même si le mandat ne visait pas le développement économique, l'étude a quand même permis de souligner la nécessité plus que jamais de limiter la dépendance aux ressources naturelles par deux avenues qui engagent les communautés dans une démarche de transformation territoriale.

- Premièrement, cela passe par des modes de gouvernance, d'exploitation, de transformation, d'utilisation et de mise en marché plus innovateurs de ces ressources. L'idée n'est pas de rompre avec l'exploitation des ressources qui demeurera longtemps encore l'activité maîtresse. Mais plus d'un demisiècle a prouvé que leur seule exploitation traditionnelle n'a pas réussi à préserver les acquis des communautés. Les innovations se cachent aussi dans les choses qu'on a tous les jours sous les yeux. Plusieurs exemples montrent que ce n'est pas une vision utopique : la première usine de co-génération du Québec à Chapais, les bois d'ingénierie chez Chantiers Chibougamau, l'argile et le bois à haute densité à Matagami et même le projet inachevé de pisciculture à Radisson.

- Deuxièmement, il y a la diversification économique. Cela étant dit, le reste repose sur les initiatives innovatrices de nature privée ou institutionnelle 
dont il faut animer l'émergence et qu'il faut soutenir, financer, mettre en valeur et accompagner professionnellement. Les secteurs de toute nature sont à privilégier : énergies, biomasse, tourisme et écotourisme, agriculture innovatrice, etc. Les saisons des idées sont ouvertes dans toutes les communautés jamésiennes.

\section{Le futur espéré des communautés jamésiennes}

Les communautés jamésiennes ont pris naissance sur la base des opportunités de travail initiées par l'État et par les grandes entreprises d'exploitation des ressources naturelles. Les populations initiales n'étaient pas des acteurs du développement, elles étaient des « facteurs de production », de la main-d'œuvre. Avec le temps, les communautés ont pris forme sur les plans politique, administratif, spatial, associatif et social. Aujourd'hui, malgré l'érosion démographique, les quatre municipalités et les localités qui composent la MBJ représentent des populations qui peuvent tenter d'influencer, à titre d'acteurs, leur propre développement.

La tâche n'est pas facile, car le développement territorial est complexe, surtout dans des petites collectivités très périphériques. D'ailleurs, le bon esprit de vivre ensemble devient encore plus important qu'ailleurs quand on est isolé et, s'il y a crise de dissension, le résultat sur les habitants peut être dramatique si on n'arrive pas à la surmonter. Il y a aussi des conjonctures exogènes et des facteurs structurels qui sont difficilement contrôlables à l'échelle de la région ou des communautés locales: mondialisation, structure démographique, concentration et financiarisation de l'économie, leviers décisionnels, éloignement géographique, etc. Il y a des modes de régulation marchande et par l'État sur lesquels les communautés ont peu ou pas du tout d'emprise.

\section{Le développement territorial de proximité se fait avec le marché, mais pas exclusivement avec lui.}

Selon un scénario et une vision dominante, il y a des forces motrices exogènes qui font paraître quasi insignifiants les discours, les stratégies et les initiatives mis en œuvre par les gens. Ils ne seraient que des pal- liatifs sans effet à long terme. Un tel scénario condamnerait les communautés ne se trouvant pas à une distance viable de grands centres et n'ayant pas de masse critique pour supporter la logique des économies d'échelle de production ou de consommation de services. Une seule solution serait alors envisageable : exploiter les ressources de manière cyclique tant qu'il y a du travail pour ensuite déménager vers les grands centres quand les opportunités seront épuisées. C'est le scénario de l'inéluctabilité que plusieurs, sinon la majorité, considèrent comme étant le réalisme.

Mais la prémisse du développement proposée ici, même si c'est difficile, part du fait que les acteurs peuvent tenter d'infléchir leur trajectoire de développement. C'est une approche volontariste, proactive et résolument pragmatique. Il devient trop facile de mettre la faute sur la concurrence étrangère, la valeur du dollar, la demande chinoise ou américaine. Ce n'est pas de la naïveté ni une vision utopique du développement. On doit tenir compte des forces exogènes et du marché, c'est une évidence. Le développement territorial de proximité se fait avec le marché, mais pas exclusivement avec lui. C'est un type de développement endo-exogène dont il s'agit ici et il repose, entre autres, sur l'innovation sociale pour améliorer une situation ou solutionner des problématiques locales et capitaliser sur les atouts à la fois endogènes et exogènes. La recette de la combinaison de ces ressources n'est pas connue, car ce serait trouver la formule du développement. Clairement, les populations ont quelque chose à y voir.

La durabilité ou non des communautés jamésiennes repose donc en partie sur sa population et sur les divers éléments du capital stratégique. Il faut d'abord que cette population se sente faire partie du territoire, en soit fière et veuille y rester pour plus que le temps d'une vie professionnelle. C'est loin d'être un acquis et c'est pourquoi travailler sur le développement social est aussi vital que le développement économique ou la gouvernance. C'est ce que l'étude a voulu mettre en évidence.

La présence des ressources naturelles d'exception et de vastes espaces, dans un contexte où l'environnement devient de plus en plus une valeur prépondérante de notre société et des autres sociétés occidentales, n'est pas quelque chose de négligeable. Qui sait si la Jamésie ne pourrait éventuellement pas devenir une destination enviée, tout comme les autres régions très 
périphériques du territoire québécois. Pour cela, il faut occuper ce territoire.

L'occupation du territoire relève d'une volonté politique nationale qui dépasse les champs d'action de la région et des communautés jamésiennes prises individuellement. Une société peut très bien décider de se fixer un objectif, qui n'est pas nécessairement économique, d'avoir des populations bien distribuées sur des espaces déjà " colonisés ». Cela peut avoir un coût comme dans bien des domaines que nous décidons collectivement comme société de financer. $\mathrm{Ce}$ coût serait à calculer, mais il ne serait pas nécessairement élevé, comparativement à d'autres décisions de financement public, entre autres parce que les populations sont somme toute peu nombreuses. Ce genre de politique pourrait offrir des mesures qui atténuent la portée des désavantages comparatifs d'éloignement et optimiser la présence des actifs d'exception qui composent certains territoires, à commencer par ses populations.

Pour terminer, il est important de prendre conscience que les éléments identifiés dans cette étude relèvent de l'interprétation de situations problématiques telles qu'illustrées par les acteurs au sein des communautés locales et de la Jamésie. Or, le développement durable des communautés passe nécessairement par l'appropriation de ces résultats d'analyse par les principaux acteurs locaux et régionaux qui pourront en tirer des réflexions pertinentes. Ils pourront aussi raffiner et pousser plus loin les dimensions que cette étude n'aurait pu mettre en évidence étant donné les limites concrètes du mandat et des moyens, particulièrement sur le plan du développement économique local et régional. Inscrit au sein d'une démarche stratégique, ce processus de réflexion mènera les acteurs à la prise en charge de leur propre développement par la mise sur pied d'initiatives et de projets de développement dans chacune des communautés locales comme au sein de la communauté régionale jamésienne.

\section{Notes et références}

1 Les auteurs sont de la firme Niska, un organisme à but non lucratif qui offre des services-conseils et de recherche en gestion du développement (www.niskacoop.com). L'article découle d'un mandat réalisé au cours de 2005-2006 pour le Comité de développement social de la Baie-James. Le rapport complet et sa synthèse sont publiés et peuvent être obtenus en communiquant au Centre régional de santé et services sociaux de la Baie James au (418) 748-3575. La référence est: Yorn, C., K. Lussier et P. Prévost (2007). Bâtir ensemble notre région, rapport intégral, Chibougamau, Centre régional de santé et de services sociaux de la Baie-James, Direction de santé publique, 66 p.

2 La subsidiarité vise la plus grande décentralisation possible des structures, à moins d'insuffisance avérée des instances inférieures. 